\title{
GENETIC AND MOLECULAR ANALYSIS OF DNA DAMAGE REPAIR AND TOLERANCE PATHWAYS
}

\author{
B. M. SUTHERLAND \\ Brookhaven National Laboratory \\ Upton, NY, 11973, USA
}

\section{Introduction}

Radiation can damage cellular components, including DNA. Organisms have developed a panoply of means of dealing with DNA damage. Some repair paths have rather narrow substrate specificity (e.g. photolyases, which act on specific pyrimidine photoproducts in a specific type (e.g., DNA) and conformation (double-stranded B conformation) of nucleic acid. Others, for example, nucleotide excision repair, deal with larger classes of damages, in this case bulky adducts in DNA.

A detailed discussion of DNA repair mechanisms is beyond the scope of this article, but one can be found in the excellent book of Friedberg et al. [1] for further detail. However, some DNA damages and paths for repair of those damages important for photobiology will be outlined below as a basis for the specific examples of genetic and molecular analysis that will be presented below.

\section{DNA Damages}

The major photoproduct formed in DNA by UV radiation is the cyclobutyl pyrimidine dimer (CPD); although [6-4] pyrimidine-pyrimidone adducts are induced at lower level, they are also significant in production of biological damage. Other pyrimidine photoproducts (e.g., hydrates) are formed by UV, but purine photoproducts are induced only at much lower levels.

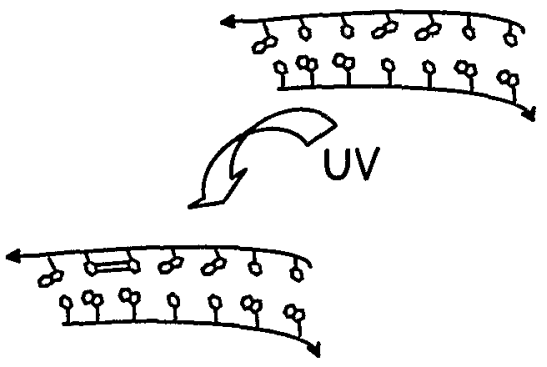

Figure 1. Induction of cyclobutyl pyrimidine dimer in DNA by UV radiation.

In addition to DNA damages formed by UV, all organisms living in an oxygen environment suffer from oxidative damage to DNA. They also carry out replication 
and other cellular metabolic processes that result in formation of single and double strand breaks. Thus, in addition to the UV-induced damages discussed above, cells must cope with oxidized purines, oxidized bases and abasic sites (sites of base loss), as well as single and double strand breaks. To deal with these UV-induced and other DNA alterations, cells have developed several distinct yet in some cases overlapping repair pathways.

DNA

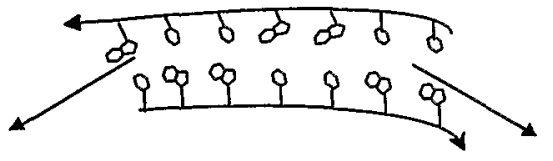

Single strand break

Oxidized bases
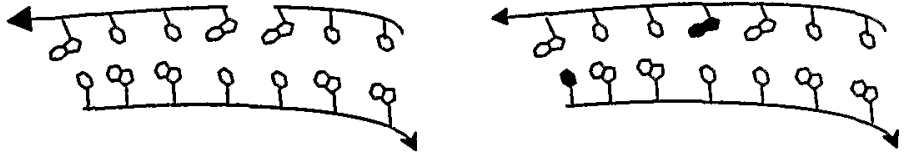

Double strand break

Abasic site
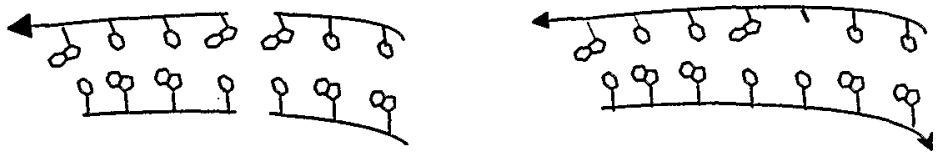

Figure 2. DNA damages induced by normal cellular metabolism in air.

\section{Photorepair}

Pyrimidine dimer photoreactivation is a one-enzyme repair path. The enzyme photolyase binds specifically to CPD in double stranded DNA, forming an enzymesubstrate complex. That complex absorbs a photon in the long UV or visible range,

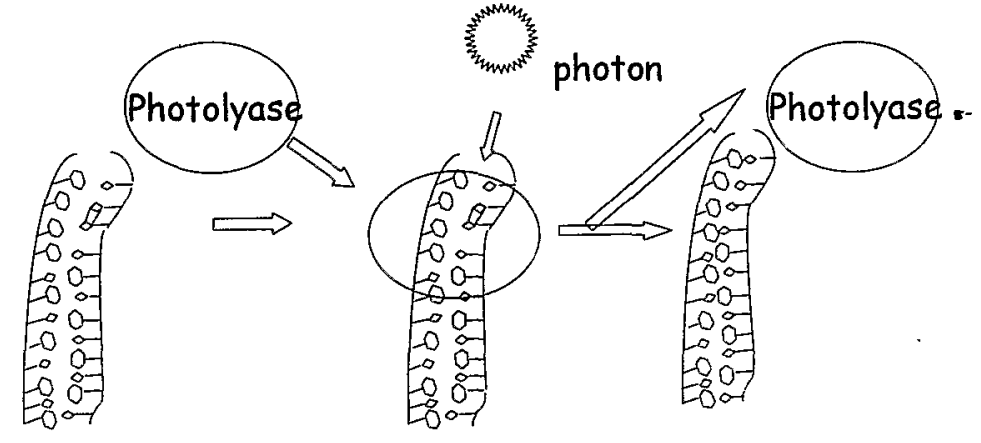

UV-damaged DNA Enzyme-substrate complex Repaired DNA

Figure 3. Mechanism of photorepair of CPD in DNA by photolyase. 
upon which the dimer is monomerized and the enzyme is released to seek another dimer. It is important to note that the formation of the enzyme-substrate complex is temperature-dependent, and also dependent on the concentration of the enzyme (number of photolyases per cell) and concentration of the substrate (CPD in DNA). The photolytic reaction depends on the wavelength and concentration (intensity) of the second substrate, the photon, but is virtually independent of temperature. Further, the enzyme-substrate complex is remarkably stable in the dark, allowing dissection of the two component reactions, i.e., enzyme-substrate complex formation and dimer photolysis.

\section{Nucleotide Excision Repair}

Nucleotide excision repair deals with a wide variety of bulky lesions in DNA, including $\mathrm{CPD}$. Several enzymes are required (including a nuclease, polymerase, ligase and other

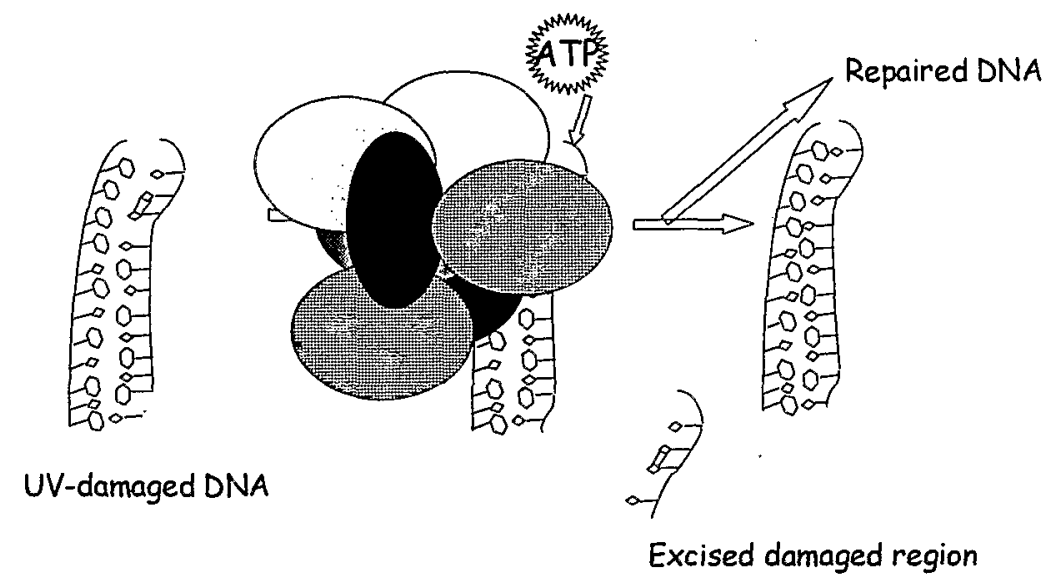

Figure 4. Repair of CPD in DNA by nucleotide excision repair.

proteins), and nucleoside triphosphates are used as an energy source. In ąddition to UV damages, this path deals with DNA alterations induced by some chemicals and even by ionizing radiation.

\section{Base Excision Repair}

Base excision repair deals with altered bases in DNA through a glycosylase activity, which removes the altered base leaving an abasic site, followed by a lyase-mediated cleavage of the phosphodiester backbone, and removal of the abasic site and restoration of the DNA by some of the proteins involved in nucleotide excision repair. The glycosylase and lyase activities may reside in the same protein or may involve separate enzymes. Abasic sites (sites of base loss) are dealt with by endonucleases that recognize and cleave the DNA at abasic sites. In human cells, an important enzyme in 
human cells for repair of abasic sites in human cells is Ape1, a 5 ' endonuclease that specifically cleaves DNA (which is then repaired by nucleotide excision repair).

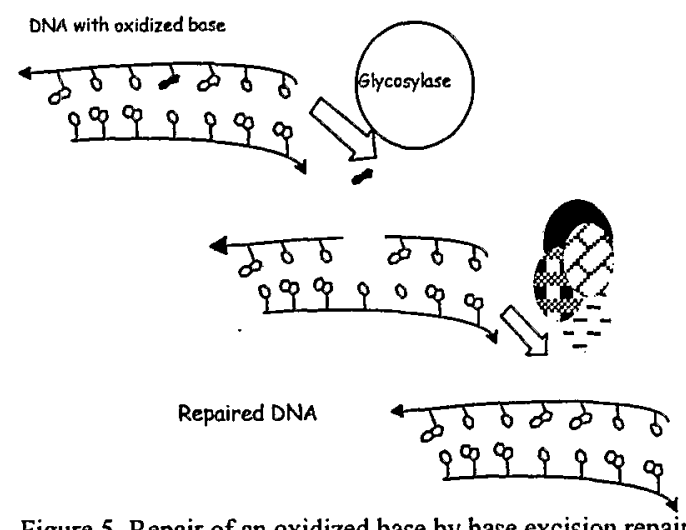

6. Principles of Molecular Dissection of DNA Repair Deficiencies

The molecular origin and the biological importance of different DNA repair paths can be explored by a variety of approaches. We consider here two approaches, in quite different systems. First, determining the molecular origin of a UV sensitive rice strain, the UV radiation sensitivity was known, but the origin of this sensitivity was not understood. There were many possible origins of the UV sensitivity, including several that were unrelated to DNA damage or repair, and the candidate genes were neither identified, cloned or sequenced $[2,3]$. Second, the sequence of a cloned DNA repair gene was examined in DNA samples from 124 normal humans. Since the biochemistry of the resulting protein was well understood, the portions of the gene whose alteration was expected to alter the function of the enzyme was known, and the potential impact of any polymorphisms could be anticipated and compared with actual activity of the altered gene product when expressed in Escherichia coli cells [4]. We will examine the approaches used in both these cases.

\section{UV Sensititive Rice: Norin 1}

Rice cultivar Norin $\mathbb{I}$ is an ancestor of many Japanese commercially-important rice strains.

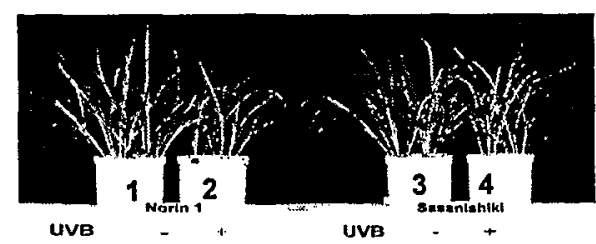

Figure 6. UV sensitive Norin 1 grown in the absence(1) and presence (2) of supplemental UVB radiation, and UV resistant Sasaniskiki grown in the absence (3) and presence (4) of UVB. 
Investigators determining the effects of UVB supplementation on various rice strains found that Norin I was exquisitely sensitive to added UVB, compared with closely related strains.

Further, the effect of supplemental UVB on other close relatives, e.g., Sasanishiki, could be ameliorated by the addition of additional white light. However, the detrimental effect of UVB could not be effectively prevented by white light supplementation. These data suggested that Norin 1 might be UV sensitive due to some deficiency in protection against UV, perhaps due to increased DNA damage or decreased DNA repair. The lack of improved UV-resistance under conditions of increased white light further suggested that Norin 1 might be deficient in light-mediated DNA repair or photorepair, the principal repair path of UV-induced DNA damage in most plants. [Background studies had already shown that Norin 1 did not differ significantly in non-repair physiological responses from its relatives.]

The first possibility was that Norin 1 was deficient in UV-protecting pigments or other cellular components, so that more damage would be formed per unit UV exposure than in its UV resistant relatives. The level of CPD per unit dose was thus determined in Norin 1 and Sasanishiki; the data clearly indicate that similar levels of CPD are formed

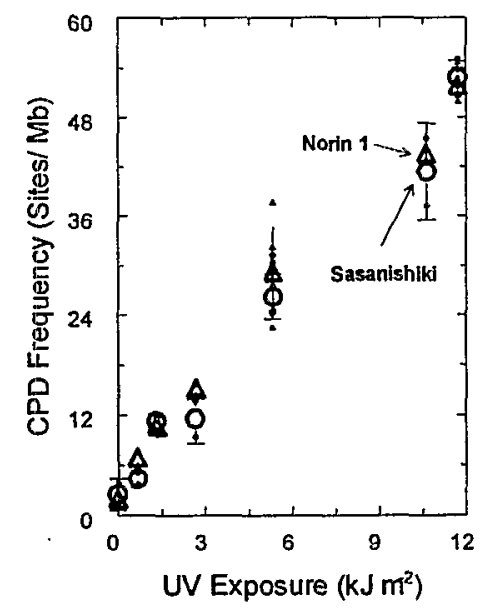

Figure 7. Equal pyrimidine dimer formation in Norin 1 and Sasanishiki rice seedlings.

in seedlings of both strains. Thus, equal exposures of UVB to these strains induce equal frequencies of DNA damage [3]. This shows that the UV sensitivity of Norin 1 did not stem from induction of more damage than in the UV resistant Sasanishiki:

Although these data suggested that Norin 1 might be defective in some repair path for UV damage, it did not indicate which repair path was affected. Hidema et al. evaluated nucleotide excision repair and photorepair in seedlings of Norin 1 and of Sasanishiki [3]. They found that UVB-irradiated seedlings of Norin I carried out photorepair much more slowly than Sasanishiki, whereas (within the time examined) the excision properties were similar. 


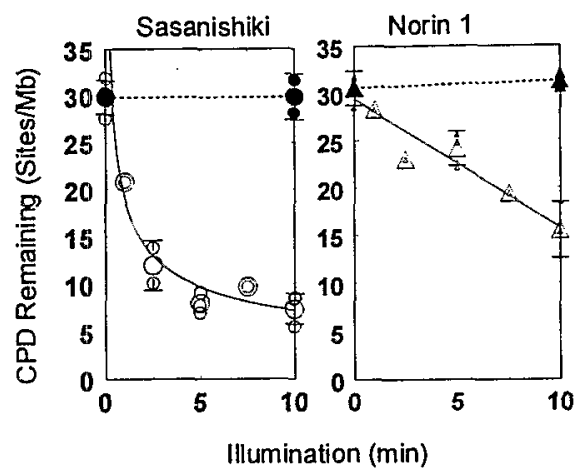

Figure 8. Photorepair (open symbols) and exision repair (closed symbols) in Sasanishiki and Norin 1

These data implicated a photorepair deficiency in Norin 1, but did not indicate if it resulted from a regulatory mutation (producing fewer normal photolyase molecules) or from a structural mutation (resulting in normal numbers of less-effective photolyases).

A powerful method for quantitating enzyme-substrate complex formation in photolyase reactions is the photoflash technique, pioneered by W. Harm, C.S. Rupert and $\mathrm{H}$. Harm [5].

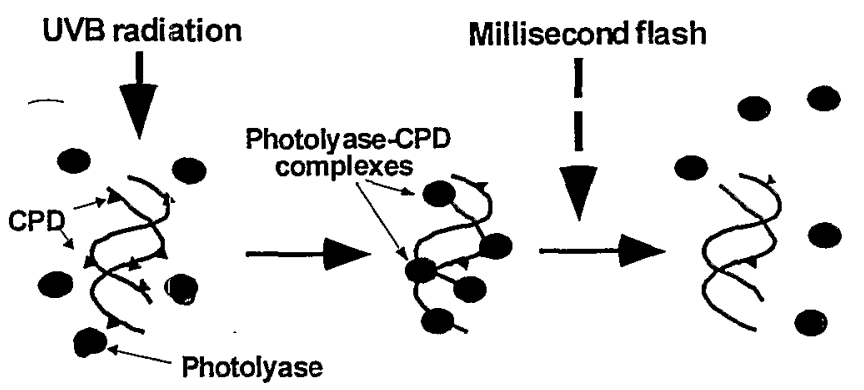

Figure 9. Principles of photoflash analysis.

In this method, DNA is irradiated to produce an excess of pyrimidine dimers. Time is allowed for all available photolyase molecules to bind to a dimer, forming an enzymesubstrate complex that is stable in the absence of light. Then one intense flash of light is applied, of sufficient intensity to photolyze all the existing photolyase-dimer complexes, and of short enough time duration that no photolyase molecules can locate and bind to a second dimer. Measurement of the number of dimers repaired then gives a direct count of the number of active enzyme-substrate complexes, and thus of the number of active photolyases.

Hidema and colleagues probed the nature of this photorepair deficiency in Norin 1 in two series of experiments using photoflash approaches to "count" the number of active photolyase molecules in a cell, to determine the rate of association of the enzyme-substrate complexes, and its stability. In the first series, the rate of association 
of the enzyme-substrate complexes and the final levels of these complexes were determined: If Norin 1 had a regulatory mutation in photolyase production, the final level of E-S complexes in Norin 1 would always be less than in Sasanishiki, no matter how long was allowed

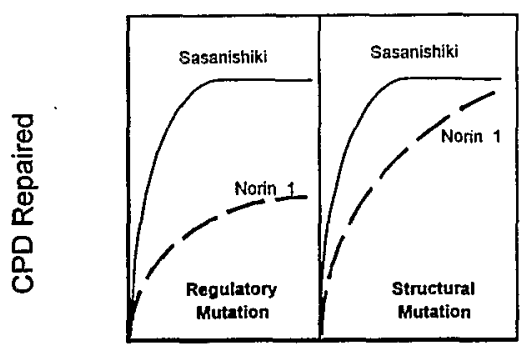

Dark Incubation Time after UV

Figure 10. Theoretical expectations for photolyase-dimer complex formation in regulatory mutants and in structural mutants of photolyase.

for complex formation. However, if it had a structural mutation in the photolyase gene, the normal levels of altered photolyase might have lowered substrate affinity (manifested by slower E-S complex formation), but given long. enough time, could form the same number of E-S complexes as its UV-resistant relative.

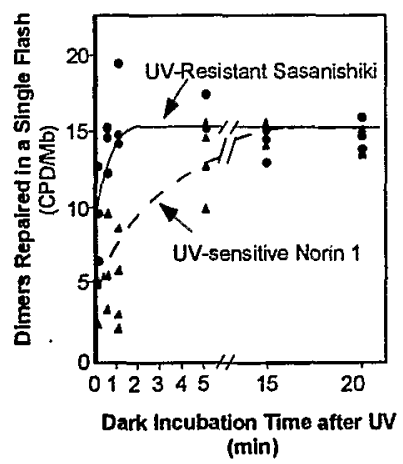

Figure 11. Photoflash analysis of photolyase-dimer complex formation in Norin 1 and Sasanishiki suggests a structural mutation in photolyase.

The data clearly showed that the latter situation was the case, prevailed, strongly suggesting that Norin 1 contained a structural mutation in photolyase that affected its function, not a regulatory mutation that decreased the number of normal photolyase molecules in each cell.

Hidema et al. wanted to confirm that this was the case, and to exclude the possibility that some non-repair factor that might slow E-S complex formation in Norin 1. One way to probe this possibility was to determine the properties of the E-S complex formed in both strains. If the slower photolyase binding (see Figure 11) was ue to an extrinsic factor, the complexes, once formed, should be similar to those formed 
in Sasanishiki. However, if the slower binding reflected lowered affinity of Norin 1 for dimers in DNA, the E-S complexes of Norin 1 might be less stable than those of Sasanishiki. Complex stability can be probed by their thermal stability: E-S complexes would be allowed to form, then challenged by incubation at a series of temperatures before administration of a photoflash. Decreased E-S stability would be reflected by

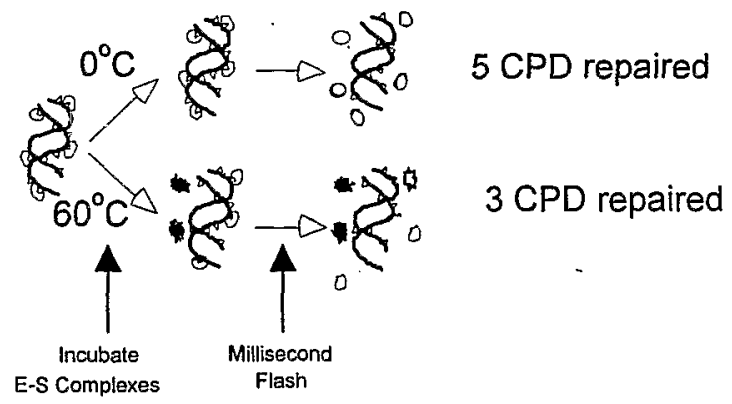

Figure 12. Measurement of photolase-dimer complex stability by photoflash analysis.

lower photorepair in the fiash reaction, whereas unaltered E-S complex stability would be reflected in similar properties of the complexes in both strains. However, since artifacts could result from heating intact seedlings, it would be preferable to use extracts of the plants.

First, it was necessary to determine whether the apparent photolyase deficiency observed in planta was observed in extracts of the seedlings. They made

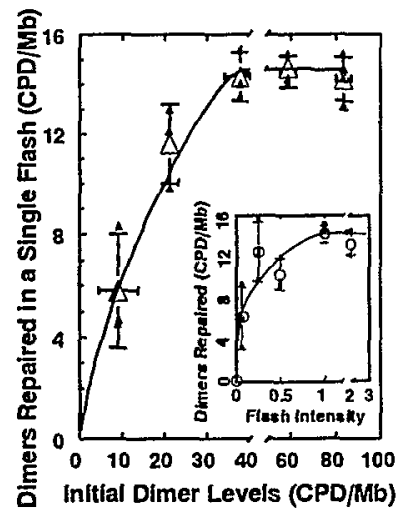

Figure 13. Photolyase deficiency in Norin 1 is observed in vivo and in vitro

extracts of seedlings of the two strains, and tested their photorepair activity in vitro, and compared the results with those for dimer repair in planta. The results are shown in Figure 13. The data show that extracts of Norin 1 seedlings also had lower photolyase activitiy than did Sasanishiki, just as is observed in the intact plants. 
They then hypothesized that if the altered Norin 1 photolyase formed stable E-S complexes more slowly, the complexes that were eventually formed might be less stable than those from Sasanishiki. They added cell extracts from Norin 1 or from Sasanishiki to UV-irradiated DNA in vitro, and incubated the mixes in the dark to allow

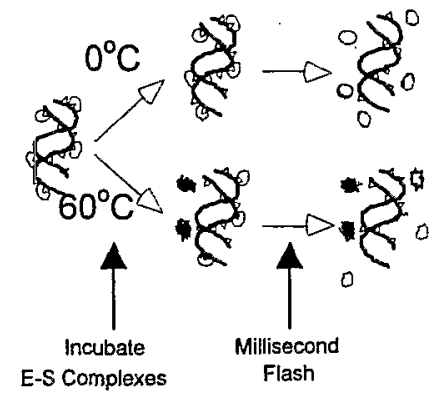

Figure 14. Measurement of photolyase-dimer complex stability by photoflash analysis.

complexes to form. They then incubated aliquots of the complexes at different temperatures, and as a function of time after complex formation, administered one saturating photoflash to allow photolysis of all existing photolyase-CPD complexes.

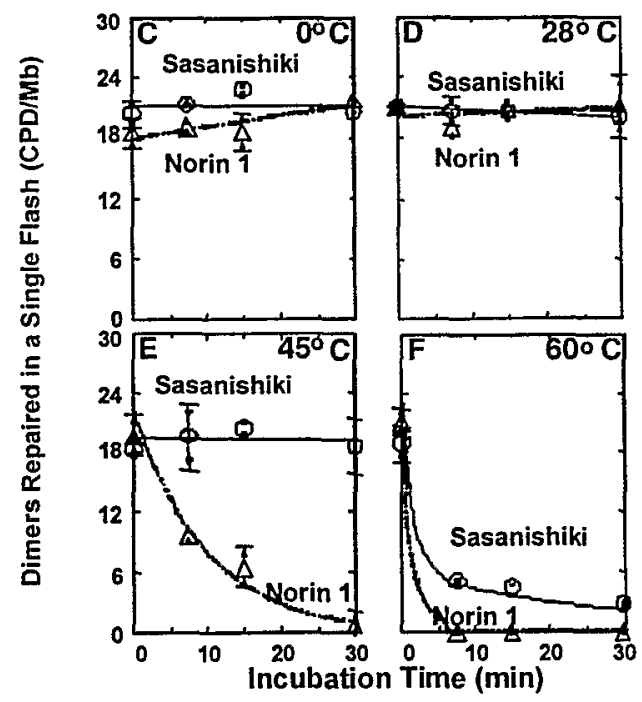

Figure 15. Determination of photolyase-dimer complex stability in extracts of Norin I and Sasanishiki at 0 , 28,45 and $600 \mathrm{C}$.

The resulting data clearly showed that the E-S complexes formed by the Norin photolyase were less thermostable than those from Sasanishiki, strongly suggesting that the Norin 1 photolyase gene has a mutation, most probably a mutation in its strucural gene that affects photolyase function. 
Photolyase genes from Norin 1 and from Sasanishiki are currently being cloned and sequence to determine the exact location(s) of any putative mutations and study their effect on photolyase function. Since photorepair is the primary DNA repair paths in plants, these studies should form the basis for determining whether other UV sensitive cultivars of rice as well as in other species contain mutations in this critical repair path for UV-induced damage.

\section{Polymorphisms of Human Ape1 Gene}

Apel is a $5^{\prime}$ endonuclease from human cells. It incises DNA at the $5^{\prime}$ side of abasic sites, producing a single strand break with a $3^{\prime}$ hydroxyl group and a 5 ' abasic terminus. It can also function at radiation-induced single strand breaks, or at sites of base loss

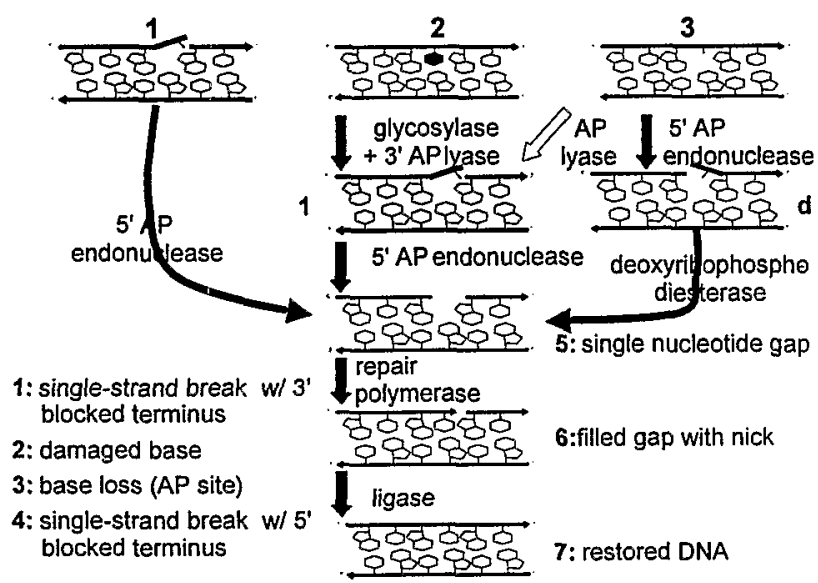

Figure 16. Action of Apel, a 5' endonuclease, in DNA repair.

resulting from base excision repair. The resulting nicked DNA is then repaired by a series of other DNA repair enzymes (See Figure 16). Since abasic sites are produced by ionizing radiation, by normal metabolism in an oxygen environment, and by glycosylases during repair of oxidized bases, such an endonuclease is essential for normal life. Mice that have been genetically engineered to lack Apel do not survive embryogenesis and decreased Ape1 activity has been observed in brain tissue of patients with amyotrophic lateral sclerosis [6]. The Apel gene has been cloned and sequenced, and the endonuclease has been overproduced, characterized, and its structure determined by X-ray crystallography. The Apel gene has two functional regions, a ref1 domain and a nuclease domain. David Wilson and Harvey

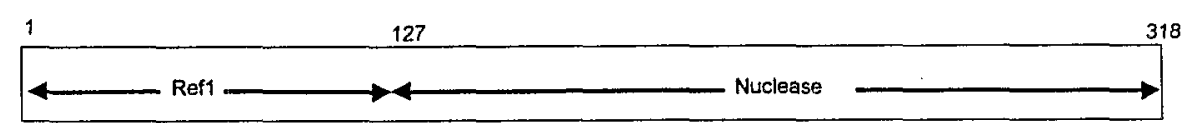

Figure 17. Human Ape I Gene with Ref1 and Nuclease Domains. 
Mohrenweiser and their groups at Lawrence Livermore National Laboratory took advantage of high throughput DNA sequencing with the knowledge base of Ape1 structure and function to look for sequence changes (polymorphisms) among normal humans in the sequence of their Apel genes (see Figure 18).

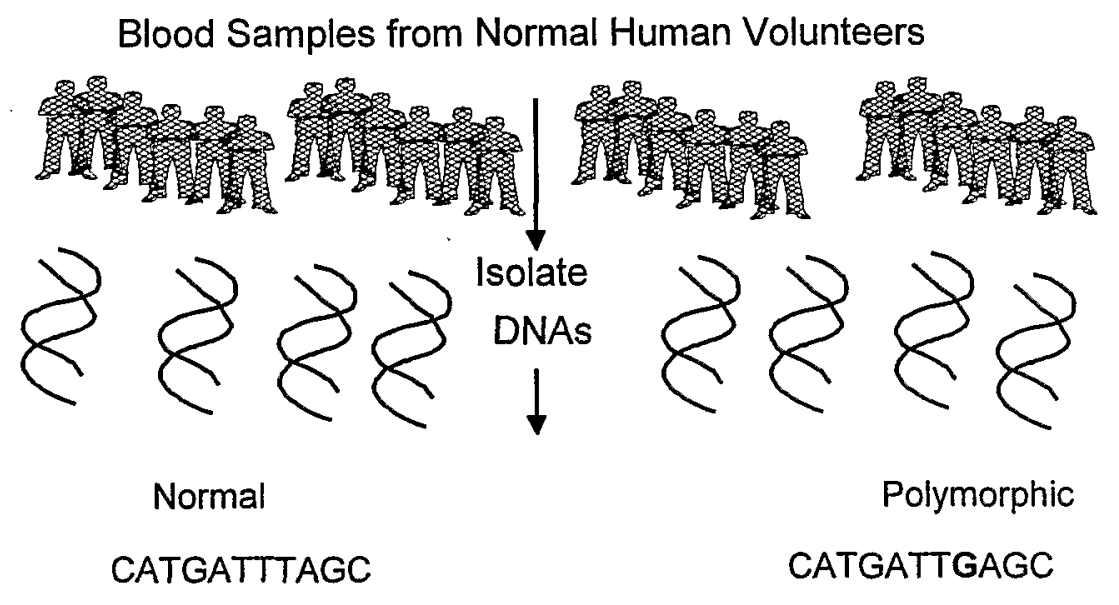

\section{Sequence Ape1 gene from each volunteer, analyze sequence}

Figure 18. Scheme for determining DNA sequence polymorphisms in Apel gene in human volunteers.

They sequenced the Apel gene from over 128 normal, unrelated people, and detected sequence changes in the repair domain of this gene from DNA in seven individuals.

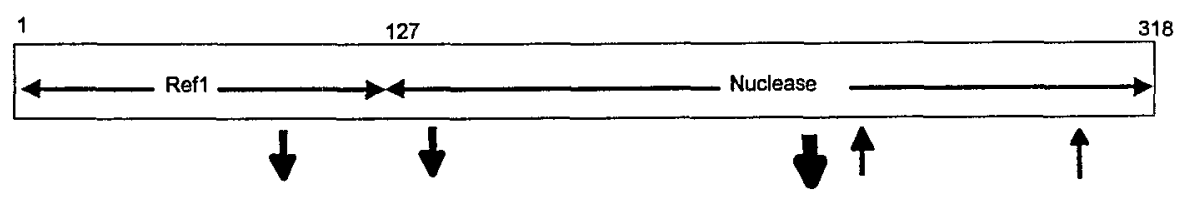

Figure 19. Human Apel gene, showing the Ref1 and nuclease domains, and locations of polymorphisms.

Of these mutations, Apel activity was decreased in 3 (shown as bold, downwardpointing arrows), of those, one was very strongly reduced (heavy bold, down-pointing arrow), increased in 2 (dotted, upward-pointing arrow) and unaffected in 2 (not shown). These mutations may affect human health, including cancer proneness.

\section{Conclusions}

Alterations of the structural genes coding for DNA repair enzymes can have severe effects on the organisms, whether a simple bacterium, or a higher plant or person. Modern biochemical and molecular biological approaches allow determination of the molecular origin of radiation sensitivity, including those resulting from deficiencies in DNA repair. 


\section{Acknowledgments}

Research supported by the Office of Biological and Environmental Research of the US Department of Energy.

\section{References}

1. Friedberg, E.C., Walker, G.C., and Siede. W. (1995) DNA repair and mutagenesis, American Society for Microbiology, Washington, D.C.

2. Hidema, J., Kumagai, T., and Sutherland, B.M. (2000) UV-sensitive Norin 1 Rice Contains Defective Cyclobutyl Pyrimidine Dimer Photolyase, The Plant Cell 12, 1569-1578.

3. Hidema, J., Kumagai, T., Sutherland, J.C., and Sutherland, B.M. (1996) Ultraviolet B-sensitive rice cultivar deficient in cyclobutyl pyrimidine dimer repair, Plant Physiology 113, 39-44.

4. Hadi, M.Z., Coleman, M.A., Fidelis, K., Mohrenweiser, H.W., and Wilson III, D.M. (2000) Functional characterization of Apel variants identified in the human population,Nucleic Acids Research 20, 38713879.

5. Harm, W., Rupert, C.S., and Harm, H. (1971) The study of photoenzymatic repair of UV lesions in DNA by flash photolysis., in A. C. Giese (eds.), Current Topics in Photobiology and hotochemistry, Academic Press, New York, pp. 279-324.

6. Kisby, G.E., Milne, J., and Sweatt, C. (1997) Evidence of reduced DNA repair in amyotrophic lateral sclerosis brain tissue, Neuroreport 8, 1337-40. 